\title{
Discriminating low frequency components from long range persistent fluctuations in daily atmospheric temperature variability
}

\author{
M. Lanfredi ${ }^{1,2}$, T. Simoniello ${ }^{1,2}$, V. Cuomo ${ }^{1}$, and M. Macchiato ${ }^{2,3}$ \\ ${ }^{1}$ Istituto di Metodologie per l'Analisi Ambientale-CNR,Tito (PZ), Italy \\ ${ }^{2}$ Consorzio Interuniversitario per la Fisica della Materia, Napoli, Italy \\ ${ }^{3}$ Dipartimento di Scienze Fisiche Università "Federico II", Napoli, Italy
}

Received: 8 December 2008 - Published in Atmos. Chem. Phys. Discuss.: 26 February 2009

Revised: 13 May 2009 - Accepted: 30 June 2009 - Published: 15 July 2009

\begin{abstract}
This study originated from recent results reported in literature, which support the existence of long-range (power-law) persistence in atmospheric temperature fluctuations on monthly and inter-annual scales. We investigated the results of Detrended Fluctuation Analysis (DFA) carried out on twenty-two historical daily time series recorded in Europe in order to evaluate the reliability of such findings in depth. More detailed inspections emphasized systematic deviations from power-law and high statistical confidence for functional form misspecification. Rigorous analyses did not support scale-free correlation as an operative concept for Climate modelling, as instead suggested in literature. In order to understand the physical implications of our results better, we designed a bivariate Markov process, parameterised on the basis of the atmospheric observational data by introducing a slow dummy variable. The time series generated by this model, analysed both in time and frequency domains, tallied with the real ones very well. They accounted for both the deceptive scaling found in literature and the correlation details enhanced by our analysis. Our results seem to evidence the presence of slow fluctuations from another climatic sub-system such as ocean, which inflates temperature variance up to several months. They advise more precise re-analyses of temperature time series before suggesting dynamical paradigms useful for Climate modelling and for the assessment of Climate Change.
\end{abstract}

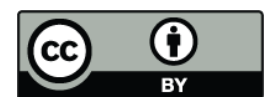

Correspondence to: $\mathrm{M}$. Lanfredi (lanfredi@imaa.cnr.it)

\section{Introduction}

The traditional stochastic approach to climate approximation is mostly based on the concept of "scale separation" (Hasselmann, 1976). The basic idea of such an approach is that climatic variability can be interpreted on the basis of a set of cycles and characteristic time scales which account for a concentration of variance in relatively well-separated spectral bands. The most direct consequence of this assumption is the well-known separation between weather and climate: fast meteorological fluctuations, which are intrinsic to the atmosphere, are mostly uncorrelated over long time periods; the accumulation of variance on low frequencies (redness) is in fact due to slow-responding climatic sub-systems such as oceans, ice cover and so on.

In the stochastic framework, meteorological variability has been traditionally explained by low-order autoregressive processes such as the paradigmatic first-order autoregressive process (AR1):

$x_{i}=a x_{i-1}+\varepsilon_{i}$

where $x_{i}$ is the meteorological variable, $a$ is the first-order autocorrelation coefficient, and $\varepsilon_{i}$ represents uncorrelated Gaussian noise (white noise). According to this model, the parameter $a$ accounts for rapid correlation decay so that the asymptotic behaviour, starting from scales of a few weeks, is uncorrelated and unpredictable: $x_{i} \sim \varepsilon_{i}$.

More recently, in the wake of the great success of empirical fractal tools devised for enhancing power-law correlation in noised and biased observational data (e.g., Peng et al., 1995; Konscielny-Bunde et al., 1998; Freeman et al., 1999; Matsoukas et al., 2000; Haggerty et al., 2002; Bunde et al., 2002; Kandelhardt et al., 2003, 2006; Blender and

Published by Copernicus Publications on behalf of the European Geosciences Union. 
Fraedrich, 2003; Varotsos et al., 2005), Fractional Gaussian Noise (FGN) (Mandelbrot and van Ness, 1968) has been suggested as a realistic model for explaining the statistical dependence of atmospheric temperature anomalies (deviations from the mean annual trend) on climatic time scales (e.g., Konscielny-Bunde et al., 1996, 1998; Govindan et al., 2001; Eichner et al., 2003; Kurnaz, 2004; Varotsos et al., 2006). The FGN correlation expresses a scale-free interdependence $c(t)=t^{-\gamma}(0<\gamma<1)$ and, for $\gamma>0.5$, the process is classified as persistent since the theoretical correlation implies a non-zero probability that disturbances survive for times as long as infinite (long-range memory). This picture is truly different from that described by the exponentially decaying correlation $c(t)=\exp (-t / \tau)$. This has a characteristic time scale $\tau$ and short memory, since the mean life time of the disturbances has a finite value $\tau$. The correlation functional form on long time scales reveals the deep dynamic nature of the interactions between atmosphere and other climatic components. Therefore, a correct assessment of this function provides useful insights for Climate modelling, also within a non-stochastic approach to the problem.

Most of the studies reported above agree on the presence of power-law persistence, which emerges after the meteorological correlation is decayed ( $\sim 20$ days) and goes on for several years. Therefore long-range memory has been suggested as an operative concept for Climate modelling (Govindan et al., 2002). Atmospheric temperature variability is often cited as an example of fractal natural process despite the contradictory results about the universality of the scaling and the dependence of the exponent on sea distance (e.g., Vyushin et al., 2004a, 2004b; Blender and Fraedrich, 2004) as well as the existence of many drawbacks in the methodologies adopted (e.g., Hu, 2001; Kantelhardt, 2001; Metzler, 2003; Mauran et al., 2004; Gao et al., 2006; Rust, 2006).

The approach of the studies on this subject is the same. In practice, they try to establish whether the asymptotic noise $\varepsilon_{i}$ in Eq. (1) is really uncorrelated or shows long-range correlation. Only two possibilities are considered in the long term: white noise or FGN. This point of view is well-expressed in the work by Király and Jánosi (2002). Since white noise (null hypothesis) is a trivial scale invariant, power-law is expected in any case; the only difference being in the numerical value of the exponents. Therefore, the actual descriptive skill of the power-law is not verified.

Nevertheless, the ability of low-order autoregressive processes to describe fast atmospheric variability lies in the concept of scale separation itself. AR1 is a reasonable model on "meteorological time-scales" where possible slower mechanisms which drive correlation do not induce remarkable variations. In other words, $\varepsilon_{i}$ represents asymptotic weather and not climate.

In order to support long-range persistence against short memory it is not sufficient to exclude white noise. Since fractal tools are extremely sensitive to any kind of non-stationary behaviour (Gao et al., 2006), this is a necessary but not suf- ficient condition and alternative correlation structures should be explicitly excluded.

Here we analysed atmospheric temperature time series in order to investigate the reliability itself of the power-law fit. We applied the Detrended Fluctuation Analysis (DFA) (Peng, 1995) to twenty-two atmospheric temperature time series recorded in Europe in order to investigate the estimated power-law persistence minutely. Some of these (Prague, St. Petersburg, Wien, Potsdam) had already been analysed in literature. We focused on patterns described by the residuals from the best fits in order to verify that they have the random character expected in the case of appropriate fitting function. Since our results indicated instead functional form misspecification for the power-law approximation, we tried to evaluate whether the empirical results were consistent with the presence of a short-memory slow component beyond meteorology. To this aim we built up a bivariate Markov process on the basis of the observational data and repeated the analyses on simulated time series, too. Our final goal was to demonstrate that a single time-scale, longer than the meteorological one, provides a satisfactory description of the sample correlation and is sufficient for tricking fractal tools by generating a deceptive fractal regime like that observed in atmospheric temperature time series.

\section{Detrended Fluctuation Analysis of temperature anomalies}

\subsection{Methods}

\subsubsection{Detrended Fluctuation Analysis}

We used the Detrended Fluctuation Analysis, which is one of the most utilized tools for minimizing externally-induced non-stationary effects describable in the form of low-order polynomials (Peng et al., 1995). We will shortly recall how this methodology works. The time series to be analysed is integrated and divided into $N$ boxes of length $n$. In each box, a least square polynomial $y_{n}(k)$, representing the trend in that particular box, is fitted to the integrated data $y(k)$. Then, the root-mean-square fluctuation $F(n)=\sqrt{\sum_{k=1}^{N}\left[y(k)-y_{n}(k)\right]^{2} / N}$ is calculated. This computation is repeated on many time-scales (box sizes) in order to characterize $F(n)$ as a function of $n$. Power-law (fractal) scaling implies a linear relationship in a log-log plot. Under such conditions fluctuations can be characterized by a scaling exponent $\alpha(\gamma=2-2 \alpha$ for FGN). The value $\alpha=0.5$ is expected for uncorrelated noise, and values $\alpha>0.5$ characterize persistent time series.

In this work we have adopted the 2nd-order detrending (DFA2) in order to minimize the effects of discontinuities and linear trends. 
Table 1. Historical time series: estimated power-law coefficients $\alpha$ obtained from DFA2 and relative $Z$-scores for random residuals probability. The considered time scale range is $20 \div 10^{4}$ days.

\begin{tabular}{llll}
\hline Station & Record & $\alpha$ & $Z$ \\
\hline Wien (AT) & $1901-2004$ & 0.63 & -97 \\
Sarajevo (BA) & $1901-2004$ & 0.55 & -98 \\
Uccle (BE) & $1833-1999$ & 0.61 & -95 \\
Basel Binningen (CH) & $1901-2004$ & 0.60 & -97 \\
Lugano (CH) & $1901-2004$ & 0.61 & -98 \\
Saentis (CH) & $1901-2004$ & 0.56 & -98 \\
Zuerich (CH) & $1901-2004$ & 0.60 & -98 \\
Prague (CZ) & $1775-2004$ & 0.63 & -96 \\
Bamberg (DE) & $1879-2001$ & 0.63 & -97 \\
Hamburg (DE) & $1891-2001$ & 0.63 & -96 \\
Hohenpeissenberg (DE) & $1879-2001$ & 0.58 & -98 \\
Potsdam (DE) & $1893-2001$ & 0.63 & -96 \\
Marseille (FR) & $1900-2002$ & 0.58 & -98 \\
Paris (FR) & $1900-2004$ & 0.59 & -96 \\
Zagreb (HR) & $1862-2001$ & 0.58 & -97 \\
Bologna (IT) & $1814-2003$ & 0.62 & -96 \\
Milan (IT) & $1763-2003$ & 0.67 & -98 \\
De Bilt (NL) & $1901-2004$ & 0.64 & -96 \\
Eelde (NL) & $1907-2004$ & 0.66 & -97 \\
Maastricht (NL) & $1906-2004$ & 0.58 & -95 \\
St. Petersburg (RU) & $1881-1998$ & 0.66 & -97 \\
Stockholm (SE) & $1756-1999$ & 0.67 & -97 \\
\hline
\end{tabular}

\subsubsection{Runs test}

The Runs Test (Bradley, 1968) determines the probability $P$ that a curve, a power-law in this specific case, deviates systematically from experimental data. A "run" is a consecutive sequence of points whose residuals are all either positive or negative. If there are $N_{+}$and $N_{-}$ points above and below the curve respectively, the number of runs $R$ in the hypothesis of fully random residuals has mean value $\left.\mu_{R}=2 N_{+} N_{-} / N_{+}+N_{-}\right)+1$ and variance $\sigma_{R}=\left(\mu_{R}-1\right)\left(\mu_{R}-2\right) /\left(N_{+} N_{-}-1\right)$. The test is performed by estimating the probability that the value of the variable $Z=\left(R-\mu_{R}\right) / \sigma_{R}$ belongs to the normal distribution. This $Z$ score expresses the divergence of the experimental result $R$ from the most probable result $\mu_{R}$ as a number of standard deviations $\sigma_{R}$ : the more negative the value of $Z$, the greater the probability of functional form misspecification due to a trend in the residuals. We wish to recall that a value $Z<-2$ is sufficient for excluding randomness with the probability $P>0.98 \%$.

\subsection{Data}

DFA was applied to twenty-two time series (see Table 1) extracted from the ECA\&D catalogue (Klein Tank et al., 2002), available at http://eca.knmi.nl/. In particular, the data recorded in Prague, St. Petersburg, Wien, and Potsdam had

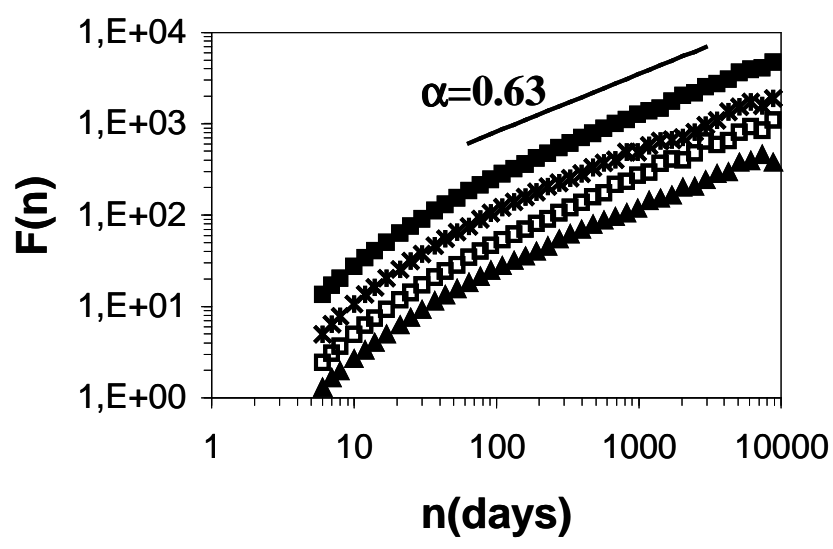

Fig. 1. DFA results for four temperature anomaly time series. From the top to the bottom: Prague (filled squares), Wien (stars), St. Petersburg (empty squares), Potsdam (triangles). Continuous line shows the theoretical power-law $n^{0.63}(\gamma=0.74)$ obtained from the power law best fits of $F(n)$ in the range $20 \div 10^{4}$ days. Plots were shifted vertically to separate the patterns.

already been analysed in literature (Konscielny-Bunde et al., 1998; Govindan et al., 2001; Eichner et al., 2003; Monetti et al., 2003; Mauran et al., 2004). By following the procedure adopted in these studies we analysed temperature anomalies (deviations form the mean annual cycle).

\section{Results}

Figure 1 shows the results of DFA2 applied to the four observational time series already analysed in literature. Plots seem to agree with slightly persistent FGN on time-scales starting from a few weeks, in complete agreement with previous studies. Temperature anomalies are basically unpredictable on such scales and possible non fractal correlations should explain very low percentages of variance. In this case, $F(n)$ is expected to describe patterns close to the trivial background power-law $n^{0.5}$. However, although small, hypothetical departures from the fractal behaviour should exhibit systematic features. In order to check the correctness of the fractal model, we focused on local details which are lost in the log$\log$ plots of $F(n)$. If the power-law fit $n^{\alpha}$ is right, the ratio $F(n) / n^{\alpha}$ is constant in theory, and its values estimated on observational data are expected to show uncorrelated variability. Instead, such a ratio is strongly patterned in a non-linear way (Fig. 2). The unfitting character of the scale invariant paradigm is especially evident on sub-annual scales where non-linearity is particularly impressive.

Although the simple naked eye inspection of our results was sufficient to draw conclusions on the misrepresentation of the power-law, we applied the Runs Test in order to quantify the goodness of fit anyway. Table 1 shows the powerlaw exponents and the values of the variable $Z$ for the whole 

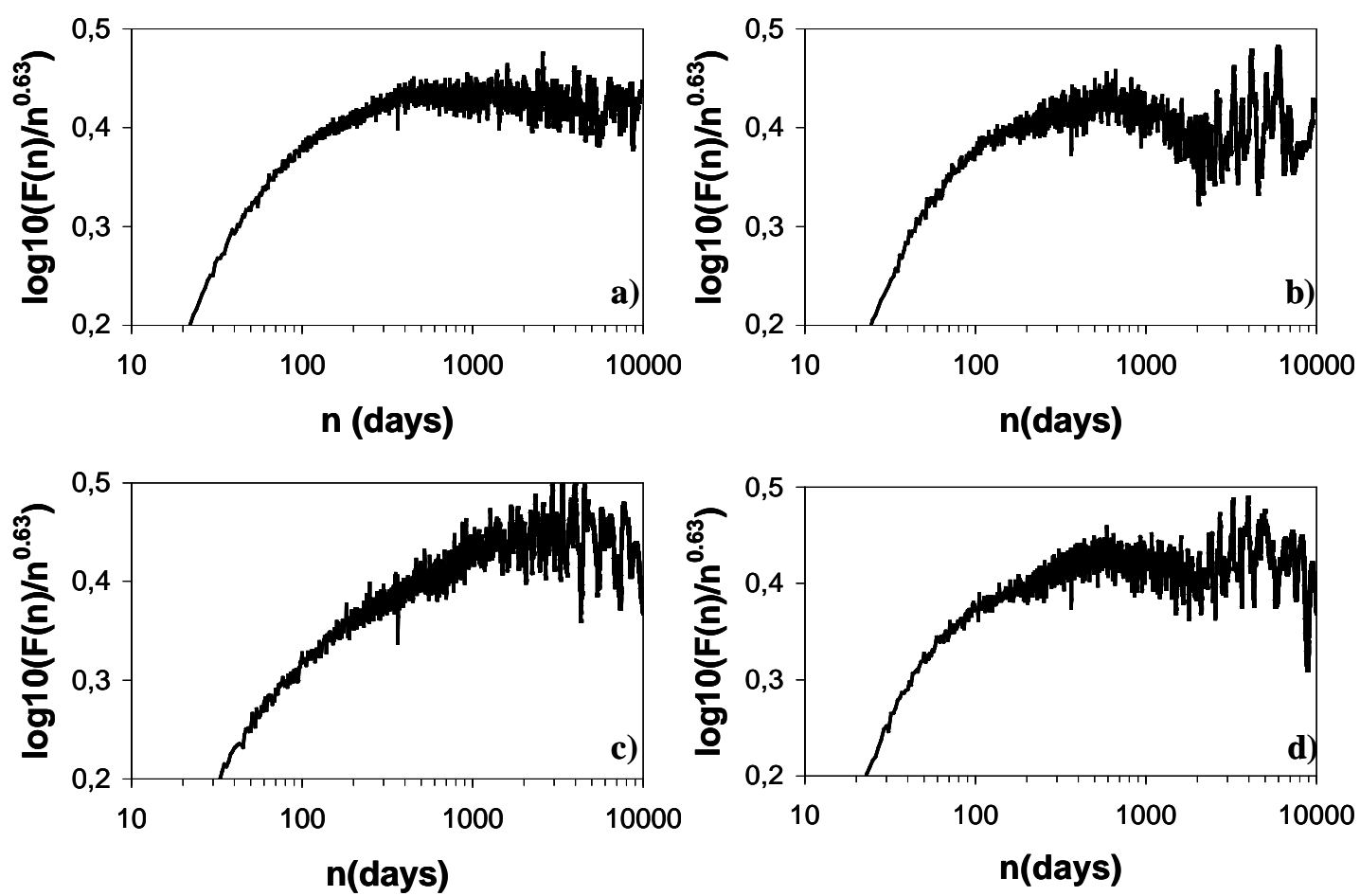

Fig. 2. Plots of the ratio $F(n) / n^{\alpha}$ in logarithmic scale for the four time series of Fig. 1: (a) Prague; (b) Wien; (c) St. Petersburg; (d) Potsdam.
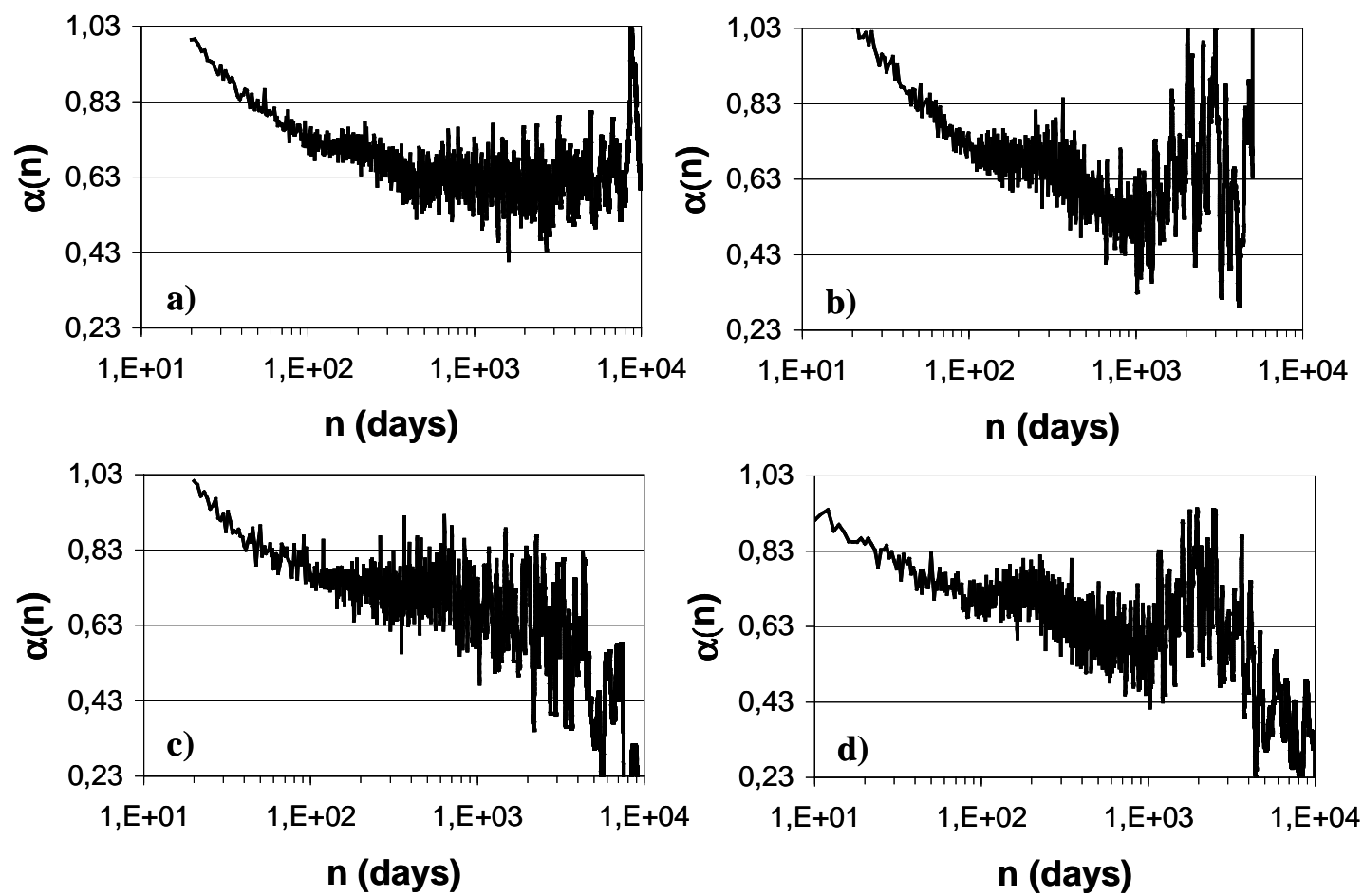

Fig. 3. Plots of the function $\alpha$ (n) for the four time series of Fig. 1: (a) Prague; (b) Wien; (c) St. Petersburg; (d) Potsdam. 
twenty-two time series. Note that all the estimated values of $Z$ are less than -90 . This means that the number of runs is placed at a distance greater than $90 \sigma_{R}$ from the mean value $\mu_{R}$ expected for random residuals; its value is always dramatically less than that expected for a reliable fitting function. If the data were randomly scattered above and below the power-law, the probability of observing so few runs in all the examined cases was $P \cong 0$. In practice, the data were fitted to the wrong equation.

As a simple alternative, it is also possible to evaluate the consistency of the fluctuation function with the scaling law $F(k n)=k^{\alpha} F(n)$. Such a relation should hold for any $k$ and $n$ in the range of the scaling regime. Figure 3 shows, as an example, the plot of $\alpha(n)=\log _{2}(F(2 n) / F(n))$ whose theoretical value should be constant for any $n$ higher than $\sim 20$ days. Once again, the results show a continuous change of the local exponent, which is close to 1 for short time-scales and decays progressively while approaching a final very irregular regime. Altogether, these findings show that the agreement between sample estimations and power-law is merely apparent since more thorough investigations enhance functional form misspecification. From a physical point of view a systematic decay of $\alpha(n)$ suggests a dependence on scale and confirms that the fractal paradigm is not suitable for describing the actual temperature variability on the scales investigated.

\section{A bivariate Markovian simulation of daily atmo- spheric temperature anomalies}

\subsection{Model development}

The simplest short memory multivariable model, which might be able to approximate atmospheric temperature variability, is the bivariate Markov process which has been suggested, as an example, to describe coupling phenomena between atmosphere and sea surface temperature (SST) (e.g, Mosedale et al., 2005):

$$
\left\{\begin{array}{l}
T_{i}=a_{11} T_{i-1}+a_{12} S_{i-1}+\sigma_{\varepsilon} \varepsilon_{i} \\
S_{i}=a_{21} T_{i-1}+a_{22} S_{i-1}+\sigma_{\eta} \eta_{i}
\end{array}\right.
$$

where $T_{i}$ is daily temperature anomaly, $\varepsilon_{i}$ and $\eta_{i}$ are normally distributed white noises, the $2 \times 2$ matrix $\left(a_{i j}\right), \sigma_{\varepsilon}$ and $\sigma_{\eta}$ are the model parameters, and $S_{i}$ denotes SST. According to the model above, the process $\zeta_{i}=a_{12} S_{i-1}+\sigma_{\varepsilon} \varepsilon_{i}$ represents innovations for $T_{i}$. On scales greater than the meteorological one, local persistence exponents could be detected since the correlation of $T_{i}$ is mainly dominated by that of $S_{i}$, which is characterized by the slower variability of the oceans.

Here we followed the suggestion provided by the model Eq. (2) and tried to build up a bivariate Markov process able to roughly reproduce temperature statistical dependence. We would like to clarify that our goal was rather general. We aimed at evaluating whether plausible mechanisms alternative to long-range memory are able to reproduce the observed

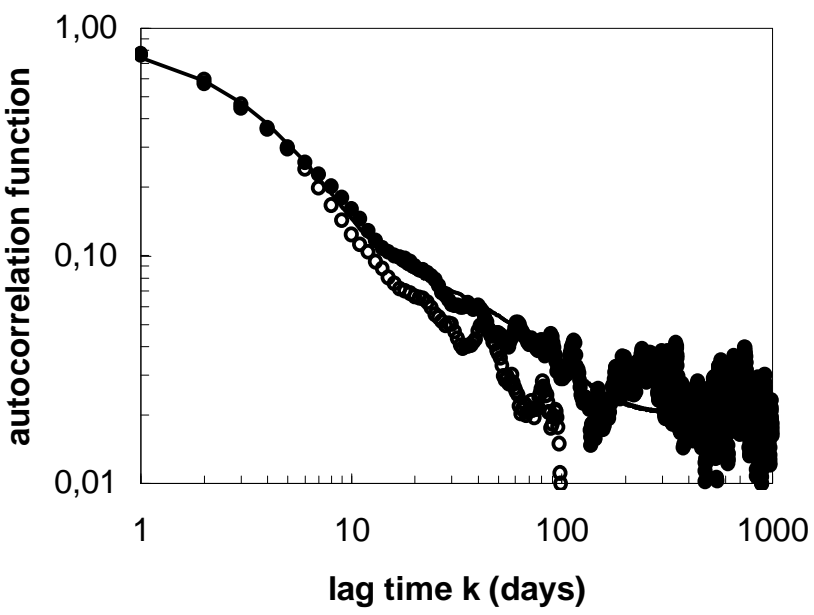

Fig. 4. Estimated autocorrelation function $\hat{\rho}(k)$ of the Prague's anomalies (filled circles); autocorrelation function $\tilde{\rho}(k)$ of the bivariate process contaminated by a linear trend (continuous line); estimated autocorrelation function $\hat{\rho}_{11}(k)$ in the absence of trend (empty circles). The $95 \%$ confidence band $(\sim 0.007)$ does not exceed the size of the symbols.

persistence. Thus, we kept the symbol $S_{i}$ to denote the twin variable of $T_{i}$ in the bivariate model, specifying that it is a (non observed) dummy variable and does not necessarily represent SST. In order to show that it is rather easy to mix up a mere low frequency component for the superposition of long range persistent fluctuations, we simplified the problem by eliminating the feedback of $T_{i}$ on $S_{i}\left(a_{21}=0\right)$ so as to handle $S_{i}$ as a truly external forcing of unknown origin. Anyway, a rough estimation of this parameter provides a value that is one/two magnitude orders less than the other "memory" parameters, just as in the modelling of Mosedale et al. (2005). Covariances $\gamma(k)_{i j}$ at any lag time $k$ were generated recursively by using scalar relations (Jenkins and Watts, 1968) which, in the hypothesis $a_{21}=0$, are:

$$
\begin{aligned}
& \gamma_{22}(k)=a_{22} \gamma_{22}(k-1) \\
& \gamma_{12}(k)=a_{22} \gamma_{12}(k-1) \quad k \geq 1 \\
& \gamma_{11}(k)=a_{11} \gamma_{11}(k-1)+a_{12} \gamma_{12}(k-1)
\end{aligned}
$$

with initial values:

$$
\begin{aligned}
& \left(1-a_{22}^{2}\right) \gamma_{22}(0)=\sigma_{\eta}^{2} \\
& \left(1-a_{11} a_{22}\right) \gamma_{12}(0)=a_{12} a_{22} \gamma_{22}(0) \\
& \left(1-a_{12}^{2}\right) \gamma_{11}(0)=\left(a_{12}^{2} \gamma_{22}(0)+2 a_{11} a_{12} \gamma_{12}(0)+\sigma_{\varepsilon}^{2}\right)
\end{aligned}
$$

The theoretical model was parameterised by fitting sample temperature autocorrelation to the function $\rho_{11}(k)=\gamma_{11}(k) / \gamma_{11}(0)$, which represents the autocorrelation of the component $T_{i}$. As a trainer for our model 


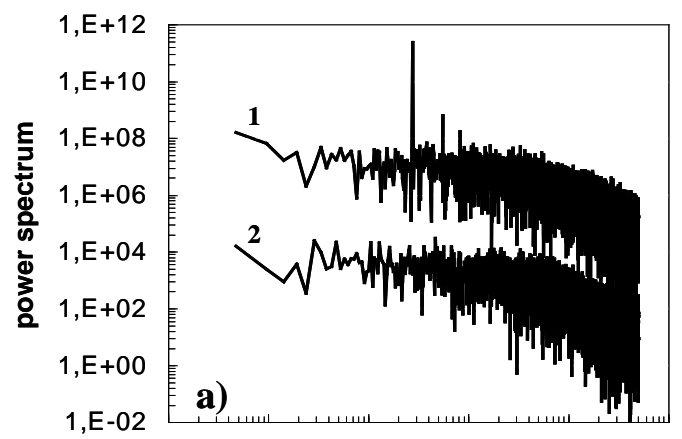

1,E-05 1,E-04 1,E-03 1,E-02 1,E-01 1,E+00

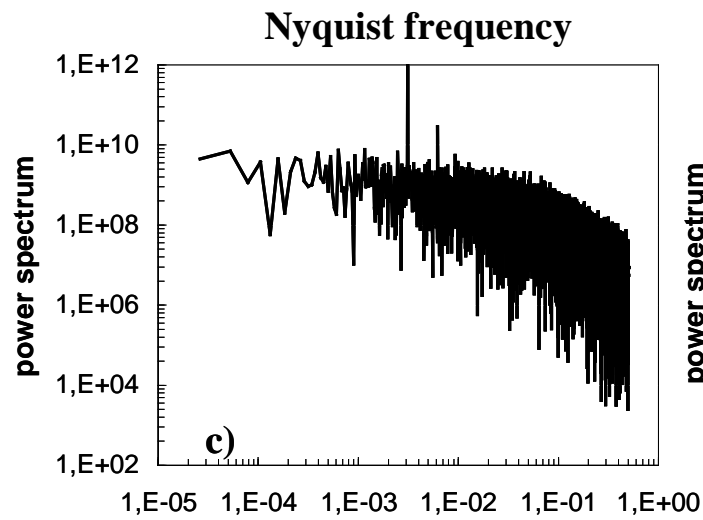

Nyquist frequency

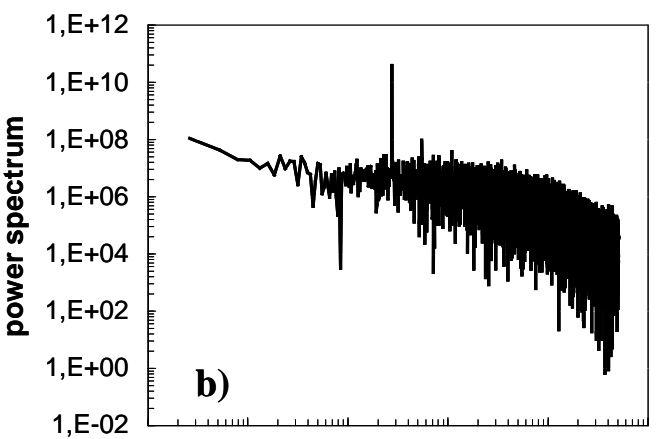

1,E-05 1,E-04 1,E-03 1,E-02 1,E-01 1,E+00

Nyquist frequency

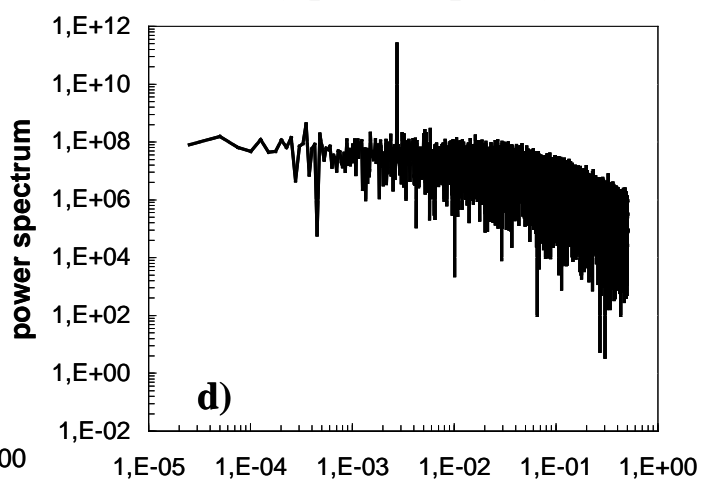

Nyquist frequency

Fig. 5. Power spectra of real temperature measures and synthetic data: (a) Prague (1) and simulated data (2); (b) Wien; (c) St. Petersburg; (d) Potsdam. Spectral peaks sign the annual and sub-annual frequencies; plots were shifted vertically.

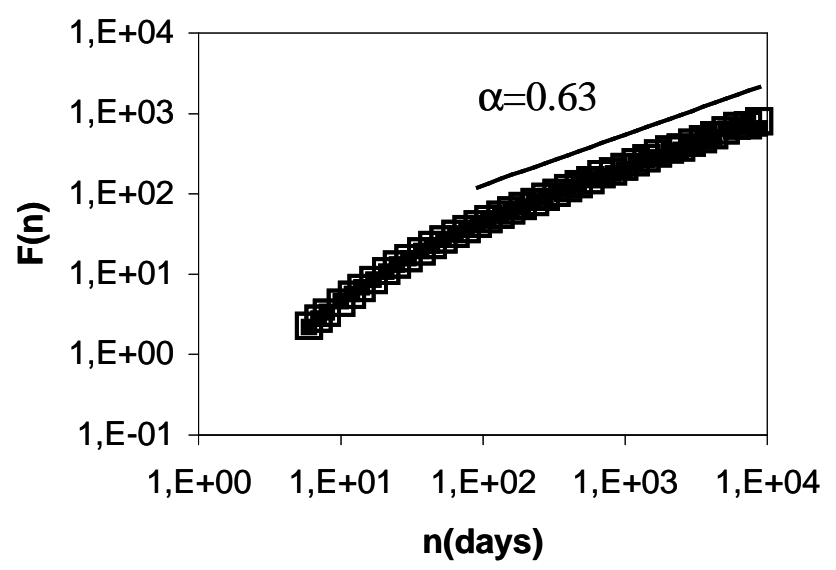

Fig. 6. Results of DFA for real (filled squares) and simulated (empty squares) anomalies. Continuous line shows the theoretical power law.

we used the time series recorded in Prague which is the longest among those illustrated in the previous Section. An increasing trend associated to urban warming (Govindan et al., 2001) is likely to be the main responsible for the slow decay of the autocorrelation $\hat{\rho}(k)$ estimated for this time series (see Fig. 4). In fact, the first autocorrelation coefficient which falls within the $95 \%$ confidence interval for zero correlation $(\sim 0.007)$ is estimated for $k=1210$ days but, if we compute $\hat{\rho}(k)$ by using only the first 100 years of the time series, this first value is estimated for $k=155$ days. Here we assumed that the effect of the trend on autocovariance for time-scales of a few years is approximately the addition of a non-zero asymptotic constant. So, we represented the fitting autocorrelation in the form $\tilde{\rho}(k)=\rho_{11}(k)+\rho_{0}$ and determined the model parameters by minimizing the root-mean-square error $E=\sqrt{\sum_{k=1}^{M}(\tilde{\rho}(k)-\rho(\hat{k}))^{2} / M}$, where $M=3650$ days (ten years). In any case, we specify that only the function $\rho_{11}(k)$ was used in the simulations.

The results of the minimizing procedure provided the best fit coefficients $\left(a_{11}=0.74, a_{12}=0.06, a_{22}=0.98, \sigma_{\varepsilon}=0.98\right.$, $\left.\sigma_{\eta}=0.39, \rho_{0}=0.01\right)$ and the autocorrelation function $\tilde{\rho}(k)$. The corresponding value of the root-mean-square error is $E_{\text {min }} \sim 0.007$, that is comparable with the $95 \%$ uncertainty on the correlation coefficients estimated on the Prague's anomalies. Thus, we considered the optimal agreement between $\tilde{\rho}(k)$ and the temperature sample autocorrelation $\hat{\rho}(k)$ quite satisfactory for our general purposes (Fig. 4) and sufficient to 

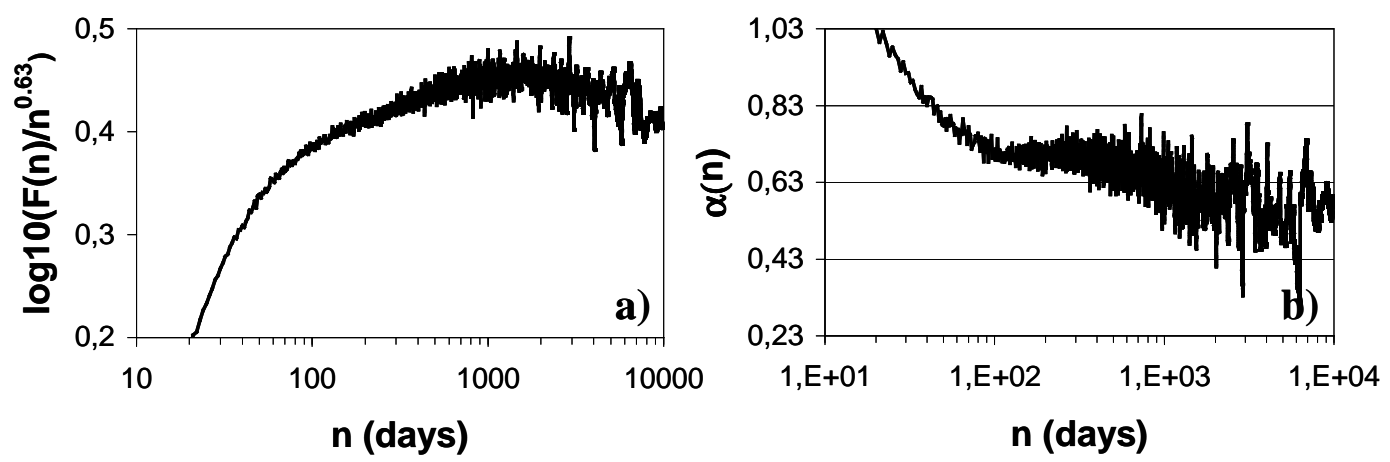

Fig. 7. As in Figs. 2 and 3 but for synthetic data: (a) $F(n) / n^{0.63}$ in logarithmic scale; (b) $\alpha(n)$.

continue with time series simulation. Obviously, the autocorrelation $\hat{\rho}_{11}(k)$ of the time series generated by the model decays faster than the total theoretical autocorrelation $\tilde{\rho}(k)$ due to the absence of the trend contribution, but the comparison of the power spectra (Fig. 5) evidences a good performance of our model despite the lack of this contribution. Note that the spectra of the real data are quite similar so that Prague's simulated time series is in a rather good agreement with the other time series too.

\subsection{Comparison between real and synthetic data}

Figure 6 shows the results of DFA2 applied to real and synthetic data. The agreement is very impressive. No evidence of any significant difference of dynamical value on the investigated scales was found in repeated Markovian simulations. Figure 7 shows the residuals from power-law and the estimates of $\alpha(n)$ for the synthetic series of Fig. 6. Also in this case the agreement with the results obtained for real data (Figs. 2 and 3) is very evident.

Simulated time series account for common properties of atmospheric temperature anomalies even if they are intrinsically not fractal. DFA is just tricked by the separation between the two scales governing $T_{i}$. Figure 8 shows this effect on the fluctuation function of the process $\zeta_{i}=a_{12} S_{i-1}+\sigma_{\varepsilon} \varepsilon_{i}$, which represents innovations for the process $T_{i}$. Due to the strong correlation of $S_{i}, \sigma_{\zeta}^{2} \sim \sigma_{\varepsilon}^{2}$ on the shortest time scales and the slope is about 0.5 , as expected for weather on uncorrelated scales. Also on long asymptotic scales such innovations are uncorrelated, but here we have $\sigma_{\zeta}^{2} \sim a_{12} \sigma_{S}^{2}+\sigma_{\varepsilon}^{2}$; in other words the explained variance is slightly greater than the initial one. This hidden increase in time also causes the increase in the fluctuation function of $T_{i}$ and the overall effect is an apparent uniform scaling. Note that also $S_{i}$ is able to produce a long lasting power-law (about 2 decades). The slope of this apparent scaling is $\alpha=1.4$, which curiously agrees with the values estimated on the same time-scales for the North Atlantic SST (Monetti et al., 2003).

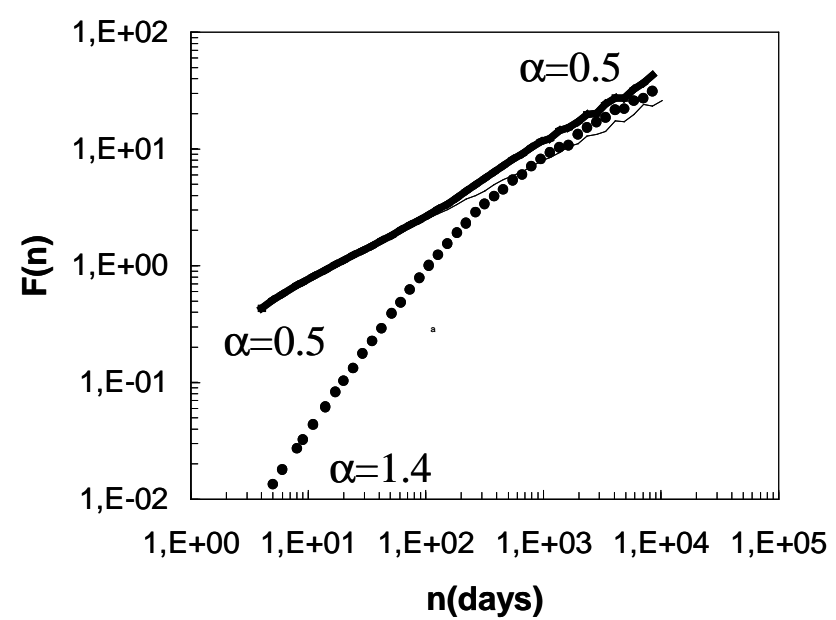

Fig. 8. Results of DFA for $\zeta_{i}$ (bold solid line), $\varepsilon_{i}$ (solid line), and $S_{i}$ (circles). $\zeta_{i}$ behaves as a white noise on short time scales and approaches a white noise with larger variance on the longest ones. This difference in variance between the extreme regimes of the innovations creates the illusory existence of persistence in temperature variability.

\section{Conclusions}

The crucial result of our work was that the existence of longrange memory mechanisms on time-scales ranging from weeks to several years has not been demonstrated yet. On such scales, where previous studies claimed the evidence of power-law correlation, persistence exponents vary with the scale instead. A simple search for straight lines in a log-log plot is not sufficient for assessing long-range memory and the reliability of the proposed correlation functional form should be explicitly tested. Moreover, a simple bivariate Markov model is able to account for the whole results, apparent scale invariance included.

More in general, the model derived in this work represents one of the many possible dynamical scenarios which can trick fractal tools. Therefore, more precise re-analyses of temperature time series are required before suggesting 
dynamical paradigms useful for Climate modelling and for the assessment of Climate Change.

Edited by: R. MacKenzie

\section{References}

Blender, R. and Fraedrich, K.: Long time memory in global warming simulations, Geophys. Res. Lett., 30(14), 1769, doi:10.1029/2003GL017666, 2003.

Blender, R. and Fraedrich K.: Comment on "Volcanic forcing improves atmosphere-ocean coupled general circulation model scaling performance", Geophys. Res. Lett., 31, L22213, doi:10.1029/2004GL020797, 2004.

Bradley, J.: Distribution-free statistical tests, Prentice-Hall, Englewood Cliffs, NJ, 1968.

Bunde, A., Schellnhuber, H.-J., and J. Kropp (Eds.): The Science of Disasters, SpringerScience, Potsdam, Germany, 2002.

Eichner, J. F., Koscielny-Bunde, E., Bunde, A., Havlin, S., and Schellnhuber, H. J.: Phys. Rev. E, 68, 046133, doi:10.1103/PhysRevE.68.046133, 2003.

Freeman, M. P., Watkins, N. W., and Riley, D. J.: Evidence for a solar wind origin of the power law burst lifetime distribution of the AE indices, Geophys. Res. Lett., 27(8), 1087-1090, doi: 10.1029/1999GL010742, 1999.

Gao, J., Tung, W.-W., Hu, J., Cao, Y., Sarshar, N., and Roychowdhury, V. P.: Assessment of long-range correlation in time series: How to avoid pitfalls, Phys. Rev. E, 73, 016117 , doi:10.1103/PhysRevE.73.016117, 2006.

Govindan, R. B., Vjushin, D., Brenner, S., Bunde, A., Havlin, S., and Schellnhuber, H.-J.: Long-range correlations and trends in global climate models: Comparison with real data, Physica A, 294(1-2), 239-248, doi:10.1016/S0378-4371(01)00110-8, 2001.

Govindan, R. B., Vyushin, D., Bunde, A., and Brenner, S.: Global Climate Models Violate Scaling of the Observed Atmospheric Variability, Phys. Rev. Lett., 89, 028501, doi:10.1103/PhysRevLett.89.028501, 2002.

Haggerty, R., Wondzell, S. M., and Johnson, M. A.: Powerlaw, residence time distribution in the hyporheic zone of a 2nd-order mountain stream, Geophys. Res. Lett., 29(13), 1640, doi:10.1029/2002GL014743, 2002.

Hasselmann, K.: Stochastic climate models, Part I, Theory, Tellus, 28, 473-485, 1976.

Hu, K., Ivanov, P. Ch., Chen, Z., Carpena, P., and Stanley, H. E.: Effect of trends on detrended fluctuation analysis, Phys. Rev. E, 64, 011114, doi:10.1103/PhysRevE.64.011114, 2001,

Jenkins, G. M. and D. Watts: Spectral Analysis and its Applications, Holden-Day, p. 332, 1968.

Kantelhardt, J. W., Koscielny-Bunde, E., Rego, H. H. A., Havlin, S., and Bunde, A.: Detecting long-range correlations with detrended fluctuation analysis, Physica A, 295, 441-454, 2001.

Kantelhardt, J. W., Havlin, S., and Ivanov, P. Ch.: Modeling transient correlations in heartbeat dynamics during sleep, Europhys. Lett., 62(2), 147-153, 2003.

Kantelhardt, J. W., Koscielny-Bunde, E., Rybski, D., Braun, P., Bunde, A., and Havlin S.: Long-term persistence and multifractality of precipitation and river runoff records, J. Geophys. Res., 111, D01106, doi:10.1029/2005JD005881, 2006.

Király, A. and Jánosi, I. M.: Stochastic modelling of daily temperature fluctuations, Phys. Rev. E, 65, 051102,
doi:10.1103/PhysRevE.65.051102, 2002.

Klein Tank, A. M. G., Wijngaard, J. B., Können, G. P., Böhm, R., et al.: Daily dataset of 20th-century surface air temperature and precipitation series for the European Climate Assessment, Int. J. Climatol., 22, 1441-1453, doi:10.1002/joc.773, 2002.

Koscielny-Bunde, E., Bunde, A., Havlin, S. and Goldreich, Y.: Analysis of daily temperature fluctuations, Physica A, 231, 393 396, 1996.

Koscielny-Bunde, E., Bunde, A., Havlin, S., Roman, H. E., Goldreich, Y. and Schellnhuber, H-J.: Indication of a Universal Persistence Law Governing Atmospheric Variability, Phys. Rev. Lett., 81(3), 729-732, 1998.

Kurnaz, M. L.: Application of Detrended Flucuation Analysis to monthly average of the maximum daily temperature to resolve different Climates, Fractals, 12(4), 365-373, 2004.

Mandelbrot, B. B. and van Ness, J. W.: Fractional Brownian motions, fractional noises and applications, SIAM Rev., 10, 422437, 1968.

Matsoukas, C., Islam, S., and Rodriguez-Iturbe, I.: Detrended fluctuation analysis of rainfall and streamflow time series, J. Geophys. Res., 105(D23), 29 165-29 172, 2000.

Mauran, D., Rust, H. W., and Timmer, J.: Tempting long-memory on the interpretation of DFA results, Nonlin. Processes Geophys., 11, 495-503, 2004,

http://www.nonlin-processes-geophys.net/11/495/2004/.

Metzler, R.: Comment on "Power-law correlations in the southernoscillation-index fluctuations characterizing El Niño", Phys. Rev. E, 67, 018201, doi:10.1103/PhysRevE.67.018201, 2003.

Monetti, R. A., Havlin, S., and Bunde, A.: Long-term persistence in the sea surface temperature fluctuations, Physica A, 320, 581589, 2003.

Mosedale, T. J., Stephenson, D. B., and Collins, M.: Atlantic Atmosphere-Ocean Interaction: A Stochastic Climate ModelBased Diagnosis, J. Climate, 18, 1086-1095, doi:10.1175/JCLI3315.1, 2005.

Peng, C. K., Havlin, S., Stanley, H. E. and Goldberger, A. L.: Quantification of scaling exponents and crossover phenomena in heartbeat time series, Chaos, 5(1), 82-87, 1995.

Rust, H.: Interactive comment on "Long-memory processes in global ozone and temperature variations" by C. Varotsos and D. Kirk-Davidoff, Atmos. Chem. Phys. Discuss., 6, S1182S1185, 2006.

Varotsos, C., Ondov, J., and Efstathiou, M.: Scaling properties of air pollution in Athens, Greece and Baltimore, Maryland, Atmos. Environ., 39, 4041-4047, 2005.

Varotsos, C. and Kirk-Davidoff, D.: Long-memory processes in ozone and temperature variations at the region $60^{\circ} \mathrm{S}-60^{\circ} \mathrm{N}$, Atmos. Chem. Phys., 6, 4093-4100, 2006, http://www.atmos-chem-phys.net/6/4093/2006/.

Vyushin, D., Zhidkov, I., Havlin, S., Bunde, A., and Brenner, S.: Volcanic forcing improves atmosphere-ocean coupled general circulation model scaling performance, Geophys. Res. Lett., 31, L10206, doi:10.1029/2004GL019499, 2004a.

Vyushin, D., Zhidkov, I., Havlin, S., Bunde, A., and Brenner, S.: Reply to the Comment on "Volcanic forcing improves atmosphere-ocean coupled general circulation model scaling performance", Geophys. Res. Lett., 31, L10206, doi:10.1029/2004GL019499, 2004b. 